\title{
Caso clínico: Diabetes Mellitus Tipo I, Pie de Charcot
}

\section{Clinical case: Diabetes Mellitus Type I, Charcot Foot}

\author{
Rosa Elvira Minchala-Urgilés ${ }^{1,3}$, María de los Ángeles Estrella-González ${ }^{1}$, Andrés Alexis Ramírez-Coronel ${ }^{1 *}$, \\ Pedro Carlos Martínez-Suárez ${ }^{1}$, David González-Pando ${ }^{5,6}$, Larry Miguel Torres-Criollo ${ }^{5}$, Diana Marcela \\ Estrella-Gonzalez ${ }^{3}$, Joaquín Sepúlveda-Aravena ${ }^{4}$ \\ ${ }^{1}$ Universidad Católica de Cuenca, Ecuador \\ ${ }^{2}$ Hospital José Carrasco Arteaga (IESS), Cuenca, Ecuador \\ ${ }^{3}$ Hospital Moreno Vázquez, Gualaceo, Cuenca, Ecuador \\ ${ }^{4}$ Centro de Educación Emocional Positiva (CEDEMOP), Talca, Chile \\ ${ }^{5}$ Grupo de Investigación PEPLAU, Ecuador \\ ${ }^{6}$ Facultad de Enfermería de Gijón, Servicio de Salud del Principado de Asturias, España \\ *andres.ramirez@ucacue.edu.ec
}

DOI: https://doi.org/10.26871/killkana_salud.v4i3.656

\begin{abstract}
Resumen
Contexto: La enfermedad de Charcot es un grupo de trastornos neuromuscula $\neg$ res que afectan, especialmente, a los nervios periféricos que conectan la medula espi $\neg$ nal y el cerebro con los músculos y los órganos sensoriales. Esta patología es de gran interés para la atención médica de los pacientes diabéticos por todas las especialidades médicas y dirigido especialmente a la atención primaria de salud. Objetivo: describir el caso de un paciente con diabetes mellitus de tipo 1 con pie de Charcot, de difícil manejo clínico y psicológico, con tendencia a la necrosis; y precisa de una resección de tejidos para resolver el problema, mediante intervención quirúrgica,. Metodología: se realizó limpieza quirúrgica del pie con aproximación de bordes más tratamiento con antibioticoterapia, que luego de 28 días de hospitalización, se va con el alta en mejores condiciones. Caso clínico: Paciente de sexo masculino, de 23 años de edad, soltero, de educación superior, acude a emergencia por presentar en el pie derecho edema grado 11 perilesional, piel del contorno descamativa, dolor intenso de tobillo, ulcera en región plantar de 4x4x4cm, con tres meses de evolución, tunelizada, exudado de mal olor y color amarillo.
\end{abstract}

Palabras clave: Diabetes Mellitus Tipo 1; Atención a la Salud; Enfermedad de Charcot-Marie-Tooth.

\begin{abstract}
Context: Charcot's disease is a group of neuromuscular disorders that affect, especially, the peripheral nerves that connect the spinal cord and the brain with the muscles and sensory organs. This pathology is of great interest for the medical care of diabetic patients by all medical specialties and directed especially to primary health care. Objective: to describe the case of a patient with type 1 diabetes mellitus with Charcot foot, difficult clinical and psychological management, with a tendency to necrosis; and requires a tissue resection to solve the problem, through surgical intervention. Methodology: surgical cleaning of the foot was performed with approximation of the edges plus treatment with antibiotic therapy, which after 28 days of hospitalization, the patient was discharged in better condition. Clinical case: 23-year-old male patient, single, with higher education, attended the emergency room due to the presence of grade II perilesional edema in the right foot, scaly skin around the contour, intense ankle pain, $4 \times 4 \times 4 \mathrm{~cm}$ ulcer in the plantar region, with three months of evolution, tunnelled, exudate with a bad smell and yellow color.
\end{abstract}

Keywords: Diabetes Mellitus Type 1, Health Care, Charcot-Marie-Tooth Disease .

\section{Introducción}

En 2014, el 8,5\% de los adultos (18 años o mayores) tenía diabetes. En 2015 fallecieron 1,6 millones de personas como consecuencia directa de la diabetes y los niveles altos de glucemia fueron la causa de otros 2,2 millones de muertes en $2012 .^{1}$
La Diabetes Mellitus es una enfermedad crónica, metabólica-degenerativa, que en la actualidad va tomando proporciones alarmantes, por los cambios asociados al estilo de vida, causando un gran impacto socio-sanitario y aparece cuando el páncreas no produce insulina suficiente o cuando el organismo no utiliza eficazmente la insulina que produce. La insulina es una hormona que regula el azúcar en la sangre. El efecto de la diabetes no controlada 
es la hiperglucemia (aumento del azúcar en la sangre), que con el tiempo daña gravemente muchos órganos y sistemas, especialmente los nervios y los vasos sanguíneos. ${ }^{1-3}$ Es un problema de salud de gran impacto sanitario y global, con una gran prevalencia, siendo una de las principales causas de ceguera, insuficiencia renal terminal, amputación de miembros inferiores y enfermedad vascular, potenciada, además, por su frecuente asociación con otros factores de riesgo de enfermedad cardiovascular como la obesidad la hipertensión arterial y la dislipidemia. ${ }^{4}$

La diabetes de tipo 1 (también llamada insulinodependiente, juvenil o de inicio en la infancia) se caracteriza por una producción deficiente de insulina y requiere la administración diaria de esta hormona. Sus síntomas consisten en: poliuria, polidipsia, polifagia, pérdida de peso, trastornos visuales y cansancio. Estos síntomas pueden aparecer de forma súbita. $^{1}$

El aumento de la esperanza de vida conlleva a un aumento de pacientes con enfermedades crónicas, entre ellas la diabetes y sus diferentes complicaciones. Dentro de las complicaciones diabéticas, la isquemia no tiene porqué presentarse en los pacientes afectados por la enfermedad y hoy en día, para el manejo de las úlceras neuroisquémicas, se dispone de una gran variedad de exploraciones, medicamentos y apósitos que intentan en la medida de lo posible evitar la amputación del miembro. ${ }^{5}$ Las úlceras y la amputación de las extremidades inferiores son la principal causa de morbilidad, discapacidad y costos para los pacientes diabéticos. ${ }^{3}$ Las neuropatías de los pies combinada con la reducción del flujo sanguíneo incrementan el riesgo de úlceras de los pies, infección y, en última instancia, amputación. ${ }^{1}$

La artropatía neuropática es uno de los trastornos degenerativos de las articulaciones de mayor interés, cada vez más comunes dentro de la patología ortopédica. Se define como una artropatía degenerativa, crónica y progresiva que afecta a una o más articulaciones periféricas y se desarrolla como resultado de la falta de percepción sensorial normal en la inervación de estas últimas. ${ }^{2}$

Los primeros síntomas de la enfermedad de pie de charcot suelen ser debilidad muscular en los pies que pueden pro $\neg$ vocar anormalidades coma el pie cavo, cavo-varo, dedos en garra y plantarflexi6n de pri $\neg$ mer metatarsiano (1 MTl). A medida que avanza la enfermedad los músculos de la extre $\neg$ midad inferior, y en ocasiones los de las manos, se debilitan. Las personas con este trastorno también pueden sufrir dolor o ardor en los pies y la parte baja de las piernas o in $\neg$ cluso disminucion de la sensibilidad al tacto y temperatura. Los síntomas pueden variar en severidad incluso en individuos de una misma familia, aunque este trastorno no suele afec $\neg$ tar a la esperanza de vida en la mayoría de pacientes. ${ }^{6}$ En ocasiones, la presentación isquémica del miembro puede ser de evolución rápida, instaurándose lesiones en el pie de difícil manejo, con dolor, infección y necrosis. $^{5}$
La exploración biomecánica del pie es imprescindible para la identificación precoz de los factores de riesgos de ulceración. ${ }^{3}$ Esta patología es de gran interés para la atención médica de los pacientes diabéticos por todas las especialidades médicas y dirigido especialmente a la atención primaria de salud. ${ }^{7}$

El objetivo de este trabajo es describir el caso de un paciente con diabetes mellitus de tipo 1 con pie de Charcot, de difícil manejo clínico y psicológico, con tendencia a la necrosis, que precisa de una resección de tejidos para resolver el problema, mediante intervención quirúrgica, registrado en el Hospital José Carrasco Arteaga, Cuenca Ecuador, 2019.

\section{Metodología}

\subsection{Representación del caso clínico}

Paciente de 23 años de edad, sexo masculino, soltero, procedente y residente en Cuenca- Ecuador, con instrucción superior. Con diagnóstico de DMT 19 años evolución, pie de charcot cinco meses de evolución, pie diabético grado III en la Escala de Wagner, neuroisquémico, con alto riesgo de amputación, paciente de alto riesgo social (véase en el Anexo 1).

Con antecedentes patológicos de hipertensión arterial, obesidad, insuficiencia respiratoria tipo I, neuropatía diabética, retinopatía diabética, mal control metabólico, trastorno emocional, diabetes mellitus tipo I desde los 14 años, coma diabético por dos ocasiones a los 15 y 18 años por falta de adherencia al tratamiento.

Motivo de consulta: paciente de sexo masculino, de 23 años de edad, acude a emergencia por presentar en el pie derecho edema grado 11 perilesional, piel del contorno descamativa, dolor intenso de tobillo, ulcera en región plantar de $4 \times 4 \times 4 \mathrm{~cm}$,con cinco meses de evolución, tunelizada, exudado de mal olor y color amarillo, pero que desde hace 5 días presenta signos de inflamación e infección, por lo que se aplica tratamiento casero sin encontrar mejoría, posterior acude a la Unidad de Pie Diabético, tras valoración se decide al ingreso a hospitalización.

Examen físico: apariencia general regular, presión arterial 130/90, hipertermia de 38.5, taquicardia 110 por minuto, $\mathrm{SO} 290 \%, \mathrm{FiO} 221 \%$, consciente, orientado en tiempo, espacio y persona, actividad psicomotora conservada, abdomen con presencia de tejido adiposo prominente, blando, depresible, timpánico a la percusión, doloroso a la palpación, presencia de zonas con signos de inflamación periumbilical por la administración de insulina, miembros inferiores con tono y fuerza muscular conservada, pie derecho presenta edema grado 11 perilesional, piel del contorno descamativa, dolor de tobillo, ulcera en región plantar de $4 \times 4 \times 4$, tunelizada, exudado de mal olor y color amarillo, signos de infección e inflamación, pulsos femoral $3 / 3$, poplíteo $3 / 3$, maleolar $2 / 3$ y pedio $2 / 3$.

Los datos de valoración en los patrones de estudio, según Marjory Gordon ${ }^{8}$ son: 
Percepción de salud: regular, con pronóstico incierto (riesgo de amputación) y con dificultades para el autocuidado.

Metabólico nutricional: paciente con sobrepeso (IMC $26.9 \%$ ), incremento de peso en los últimos años, se alimenta de dieta hipercalórica, hiperproteíca.

Actividad y ejercicio: en reposo relativo, taquipnea 30 por minuto, tono y fuerza muscular conservada, de miembro inferior derecho poco disminuida, marcha inestable por presencia de lesión en el pie.

Autopercepción: falta de motivación, ansiedad, depresión, problemas de autoestima y de personalidad.

Relaciones: soltero, conflicto con familiares, apoyo insuficiente de familiares, estudiante de instrucción superior, fuma 10 cigarrillos por semana e ingiere alcohol desde los 15 años de manera ocasional.

Tolerancia al estrés: con dificultad de afrontar la crisis y el estrés, dependiente familiar.

Valores y creencias: actitud ante el sufrimiento y dolor, preocupación por su enfermedad.

Exámenes complementarios:

- Examen de pie derecho en tres posiciones, resonancia magnética.

- Se descarta lesiones estenóticas y oclusivas de vasos distales y trombosis venosa profunda, mediante un ecodoppler.

- Laboratorio: glicemia $383 \mathrm{mg} / \mathrm{dl}$, urea $45.4 \mathrm{mg} / \mathrm{dl}$, neutrófilos $58.8 \%$, linfocitos $31.5 \%$, hematocrito $37 \%$, hemoglobina $12.4 \%$, HbA1c $12.4 \%$. se administra Insulina lantus 30-0-0, Insulina Rápida 10-20-10

\subsection{Evolución}

Paciente desde los 14 años diagnosticado de diabetes mellitus tipo I, coma diabético por dos ocasiones a los 15 años y 18 años, hace 3 años presenta cambios tróficos en los pies como: edema, hiperqueratosis, hormigueo, hace 1 año camina descalzo provocándose una ulcera de difícil manejo (3 meses), cada año presenta ulcera por mal cuidado de los pies. Paciente ingresa el 9 de abril y es dado de alta el 28 de mayo del 2019 (50 días de hospitalización). El 24 de abril ingresa a la Unidad de cuidados intensivos por edema agudo de pulmón/neumonía, por sobrecarga de líquidos, insuficiencia respiratoria tipo I, SO2 89\%, FiO2 $36 \%$, ingresa a ventilación mecánica, el 30 de abril es dado de alta a piso con cuadro recuperado. Egresa del hospital el 28 de mayo luego de recibir curaciones y mejoría de la ulcera en el pie. Cuatro meses después, (el 20 de septiembre) reingresa por presentar temperatura de 38,5 grados, malestar general, dificultad a la deambulación, edema e inflamación en la cara dorsal y plantar del pie y tobillo, dolor de moderada intensidad, se observa colección tabicada de aproximadamente $22 \times 16 \mathrm{~cm}$ entre el tercer y quinto dedo de la región metatarsiana, por lo que se realiza destechamiento plantar más limpieza quirúrgica (Anexo 2) del pie derecho, con aproximación de bordes (Anexo 3), colocándose férula de yeso posterior, se complementa con tratamiento famacológico: losartan 100mg cada día, simvastatina 20mg cada día, Insulina Lantus 36 UI cada día, Insulina Novo rapid 6UI predesayuno y prealmuerzo, 10UI premerienda, imipenem 500mg cada 6 horas por 10 días, linezolid 600mg cada 12 horas por 10 días. Paciente es dado de alta luego de 12 días de hospitalización, con educación en autocuidado y controles por consulta externa.

Paciente con trastornos de adaptación al medio y estrés grave, ha evolucionado favorablemente, luego del alta de cuidados intensivos, recibe capacitación y ayuda psicológica todos los días, con material de apoyo, para que el paciente desarrolle habilidades y destrezas en el manejo de la enfermedad.

\section{Desarrollo y Discusión}

La diabetes mellitus tipo 1 (DMT1) es una enfermedad autoinmune cuya etiología implica predisposición genética y factores ambientales. Los polimorfismos de algunos genes se encuentran entre los factores genéticos más importantes que influyen en la autoinmunidad. ${ }^{9}$

De acuerdo con la Asociación Americana de la Diabetes, más de 25 millones de personas en Estados Unidos tienen esta enfermedad, alrededor de $8 \%$ de la población también la padece y no está diagnosticada. De 60-70\% de las personas con diabetes desarrollan daño en los nervios periféricos y hasta $29 \%$ de estos pacientes pueden presentar artropatía de Charcot. En la mayoría de los casos esta complicación es tardía, por lo general aparece después de que el paciente ha padecido diabetes durante un largo período. ${ }^{10}$

En Ecuador, según la encuesta ENSANUT, la prevalencia de diabetes en la población de 10 a 59 años es de $1.7 \%$, demuestra que la prevalencia de la obesidad está aumentando en todos los grupos de edad; 3 de cada 10 niños en edad escolar presenta sobrepeso y obesidad; 2 de cada 3 ecuatorianos entre los 19 y 59 años tiene sobrepeso y obesidad, lo que constituye un serio problema de salud pública. ${ }^{11}$

La enfermedad de Charcot es un grupo de trastornos neuromuscula $\neg$ res que afectan fundamentalmente a los nervios periféricos que conectan la medula espi $\neg$ nal y el cerebro con los músculos y los órganos sensoriales. Los primeros síntomas suelen ser debilidad muscular en los pies que pueden pro $\neg$ vocar anormalidades como el pie cavo, cavo-varo, dedos en garra y plantarflexión de pri $\neg$ mer metatarsiano. ${ }^{6}$

Loupa, reporta un caso de un paciente joven de 28 años, diabetes tipo I, 12 años de evolución, HbA1c=14.9\%, con pie diabético que fue tratado con éxito al momento del diagnóstico con descarga bilateral de aire. Desafortunadamente, debido a la depresión y el incumplimiento, la enfermedad progresó a lesiones graves y permanentes más adelante. ${ }^{12}$

Sánchez, 2018, ${ }^{3}$ presenta a un paciente con diabetes tipo I con amputación transtibial por vasculopatía diabética, que presentaba hiperqueratosis en primer y quinto metatarsianos en zona plantar así como alteraciones tanto en la sensibilidad superficial como profunda, un caso es de gran inte- 
rés para la atención médica de los pacientes diabéticos por todas las especialidades médicas y dirigido especialmente a la atención primaria de salud Vallés Gamboa $\mathrm{AS},{ }^{7}$ analiza un paciente de 21 años, con diabetes mellitus de tipol con pie de Charcot, por presentar una artropatía neuropática de Charcot, en el estadio tres de Eichenholtz, con 12 años de evolución, un mal control metabólico, hemodiálisis por insuficiencia renal y en su posterior evolución comenzó a presentar pericarditis urémica con fallo cardiaco y fallecimiento posterior.

Un estudio en Perú, ${ }^{13}$ donde se incluyeron 330 pacientes con diabetes, la prevalencia de neuropatía periférica fue de $44,2 \%$ y analiza un caso de paciente de 38 años con DM tipo I por hiperglucemia con cetosis, poliuria y polidipsia intensa de 3 semanas de evolución, acompañado de la pérdida de $12 \mathrm{Kg}$ en el último año, glucemia $340 \mathrm{mg} / \mathrm{dl}$, IMC: $17 \mathrm{Kg} / \mathrm{m}^{2}$, tensión arterial $145 / 95 \mathrm{mmHg}$ y frecuencia cardíaca de 100 latidos por minuto, hemoglobina glucosilada al debut fue de 13,4\%. Datos importantes reflejados en relación al estudio de caso que se presenta con el paciente de 21 años, con presión arterial 130/90, hipertermia de 38.5, taquicardia 110 por minuto, glicemia $383 \mathrm{mg} / \mathrm{dl}, \mathrm{HbA} 1 \mathrm{c}$ $12.4 \%$, con complicaciones clínicas y psicológicas.

El tratamiento se centra principalmente en la interrupción del proceso inflamatorio y el alivio del dolor utilizando dispositivos de descarga de pies. En casos más graves, puede ser necesaria una intervención quirúrgica. Además, el uso de plantillas personalizadas y zapatos ortopédicos personalizados es obligatorio. ${ }^{12}$

La educación diabetológica es el proceso de proporcionar al paciente un conocimiento de las habilidades necesarias para llevar a cabo el autocontrol y el manejo de las crisis, y para realizar cambios en el estilo de vida. Como resultado de este proceso, el paciente conocerá y será un participante activo en su enfermedad. ${ }^{4}$ La relación médico paciente es fundamental para lograr la adhesión del mismo al tratamiento, del que la información y la educación forman parte. La mayor posibilidad de curación del pie de Charcot yace en el temprano reconocimiento de la enfermedad y en el tratamiento conservador con correcta inmovilización, en la mayoría de los casos. ${ }^{14}$

La orientación del equipo multidisciplinar es indispensable en el desarrollo del autocuidado, favoreciendo la independencia y la adaptación del paciente a sus actividades diarias. El personal profesional de salud responsable del cuidado debe tener conocimiento especial sobre el manejo y educación al paciente-familia y al mismo tiempo adaptarlo al entorno social. ${ }^{15}$ El equipo multidisciplinar debe estar conformado por podólogo, traumatólogo, internista, cirujano vascular, endocrinólogo y enfermeras capacitadas en la especialidad.

\section{Conclusiones}

Paciente con 28 días de hospitalización, se va con el alta en mejores condiciones, se educa sobre el manejo adecuado de su enfermedad, propuesta por parte del paciente elaborar un documental para compartir con los otros pacientes. Se sugiere seguimiento en clínica de heridas y unidad de pie diabético.

Dada la magnitud de la población en riesgo de padecer la enfermedad, resulta imposible que todos los pacientes sean atendidos por el médico especialista, por lo tanto, es necesario que los de atención primaria cuenten con herramientas, sencillas y actualizadas que les permitan el abordaje (educacion), la evaluación y el tratamiento del paciente diabético, así como decidir cuándo deberían referirlo al especialista (endocrinólogo, oftalmólogo, nefrólogo etc.). Es importante la inspección minuciosa de los pies todos los días, al igual que el control glucémico periódico.

\section{Anexos}

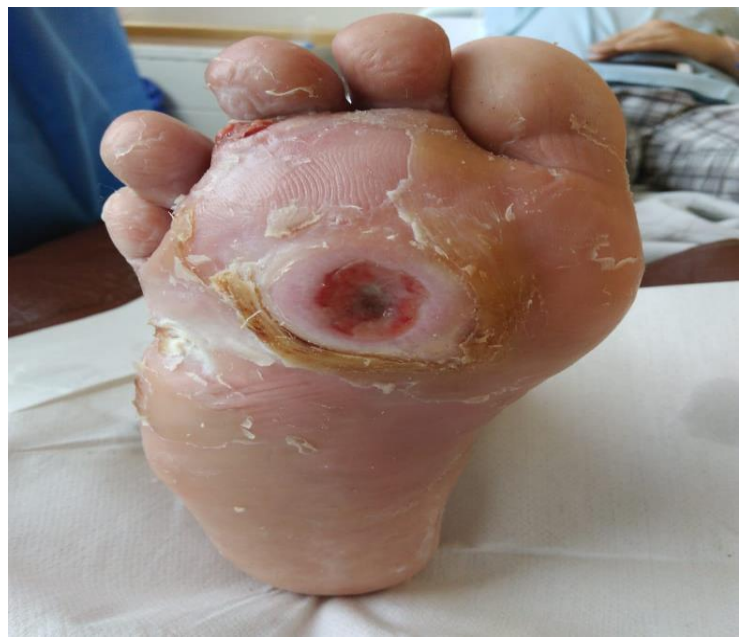

Anexo 1. Motivo de ingreso

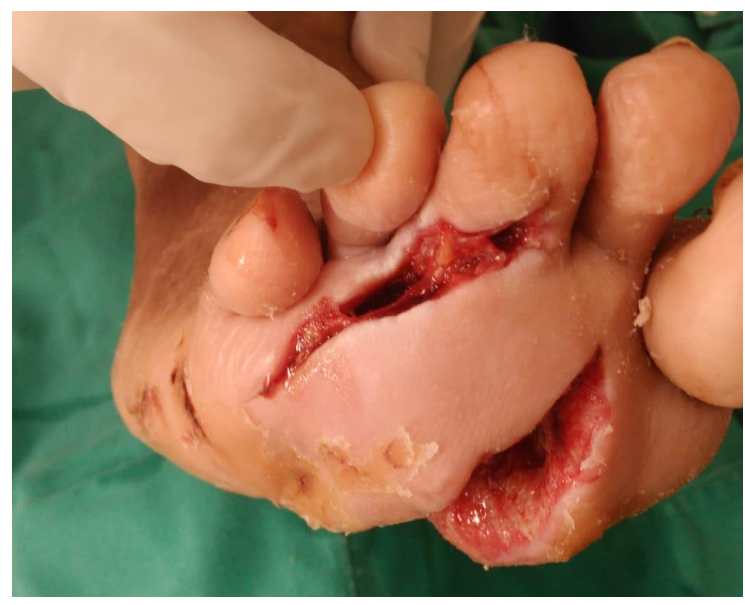

Anexo 2.Limpieza quirúrgica 


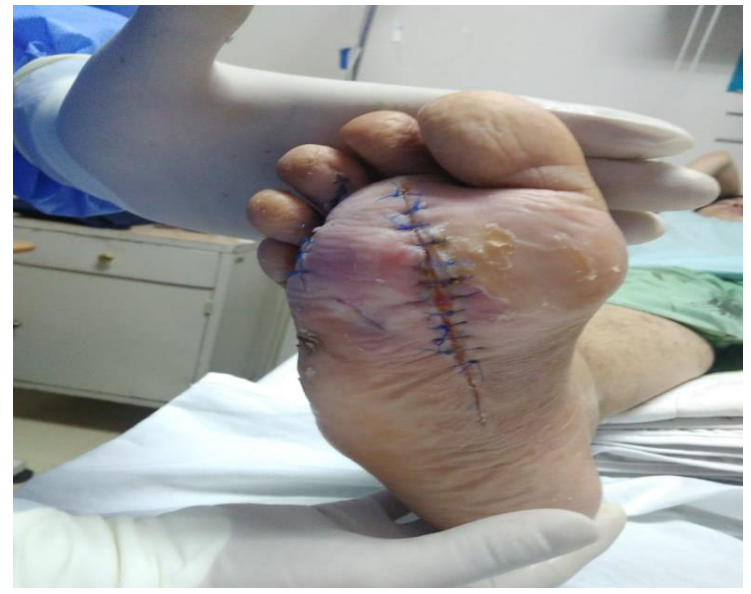

Anexo 3.Aproximación de bordes

\section{Fuente de Financiamiento}

Este estudio es autofinanciado.

\section{Conflicto de Intereses}

No existen conflictos personales, profesionales, financieroso de otro tipo.

\section{Consentimiento Informado}

Los autores cuentan con el consentimiento informado de los pacientes para la investigación, la publicación del caso y sus imágenes.

\section{Referencias Bibliográficas}

1. Organización Mundial de la Salud. Diabetes [Internet]. Ginebra: OMS; 2018 Disponible en: https://www.who.int/ es/news-room/fact-sheets/detail/diabetes

2. López E, Parra, Vázquez J. La neuroartropatía de Charcot en el pie diabético. Rev. Acta Ortop Mex. 2016; 30(1).

3. Sánchez M, Alcazar E. Análisis cinemático de tobillo en plano sagital de paciente diabético con amputación transtibial: A propósito de un caso. Rev Cuest de fisioter. 2018;47(3): 224-232.

4. Gil LE, Sil MJ, Domínguez ER, Torres L, Medina JH. Guía de práctica clínica. Diagnóstico y tratamiento de la diabetes mellitus tipo 2. Rev Méd del Inst Mexi del Seg Soc. 2013;51(1): 1-16.

5. Fernández VL, Llatas FP, Pujalte BF, Talamantes CS. Manejo del paciente diabético con úlcera neuroisquémica:
A propósito de un caso. Enferm Dermat. 2012;6(16):3438.

6. Sarroca N, Gallart J, González D, Valero J. El pie en la enfermedad Charcot Marie Tooth: A proposito de un caso. 2017;38(2): 22-29.

7. Vallés AS, Velázquez W, Osorio CM. Diabetes mellitus de tipo 1 y pie de charcot en el tercer estadio de Eichenholtz. Rev cubana. 2015;16(1).

8. Herdman T. Kamitsuru S. NANDA Internacional; Diagnósticos Enfermeros, definiciones y clasificación. Edición Hispanoamericana Elsevier; 2015.

9. Samahy M, Toaima D, Elshami Y. Effect of Estrogen Receptor- alpha gene polymorphism (IVS1-397 T>C) on microvascular complications of Type 1 Diabetes Mellitus. Rev. Curr Diabetes. 2019.

10. Morrison WB, Shortt CP. Ting AYI. Imaging of the Charcot foot. Brooklandville: Data Trace Publishing Company; 2010 .

11. Naranjo Y. La diabetes mellitus: un reto para la Salud Pública. Rev Finl. 2016; 6(1): 1-2.

12. Loupa CV, Meimeti E, Kokas A, Voyatzoglou ED, Donou A. An atypical and bilateral presentation of Charcot foot disease. BMC Endoc Disord. 2019;19(1):1-5.

13. Oliveros L, Egaña N, Aramburu M, Yoldi A, Goena Iglesias M. Debut de diabetes mellitus en paciente joven: diabetes tipo 1. Rev. Endoc y Nut. 2012;59(4):275-276.

14. Schinca N, Alvarez J. Artropatía Neuropática o Pie de Charcot. Rev. Biomed. 2012;7(1): 44-50.

15. Minchala R, EstrellaM. Caso Clínico: Abordaje de Ostomías. Rev. Méd HJCA; 2017;9(2): 181-185.

Recibido: 3 de febrero de 2020

Aceptado: 16 de agosto de 2020 
